\title{
Comparison of AHP and Monte Carlo AHP Under Different Levels of Uncertainty
}

\author{
Niam Yaraghi, Pooya Tabesh, Peiqiu Guan, and Jun Zhuang
}

\begin{abstract}
Despite the extensive application of Monte Carlo analytic hierarchy process (MCAHP) in various fields of decision making, its performance has not been compared with the classic analytic hierarchy process (AHP). Both of these methods are heavily affected by individual or group preferences and thus provide subjective rankings. Since the mere difference between their results does not necessarily warrant the superiority of one against the other, a reliable and robust ranking of alternatives should be available as a comparison basis so that the results of these two methods can be evaluated. In this paper, we use a simulation approach to compare the results of AHP with MCAHP under different levels of uncertainty. We validate our simulation results by comparing the performance of these two alternatives against a real world and reliable ranking of blogs. Our simulation results show that as long as the variation in different pairwise comparisons is less than 0.24 , the performance of AHP is not statistically different from the performance of MCAHP. When the uncertainty in terms of variation grows beyond 0.24 , MCAHP provides more precise rankings. The findings of this research add to the current body of knowledge in the multicriteria decision analysis as well as Information Systems literature and provide insights for managerial applications of these techniques.
\end{abstract}

Index Terms-Analytic hierarchy process (AHP), Monte Carlo analytic hierarchy process (MCAHP), simulation.

\section{INTRODUCTION}

$\mathbf{S}$ INCE ITS introduction, analytic hierarchy process (AHP) has become widely accepted by professionals and scholars as a reliable, yet easy to use, multicriteria decision-making (MCDM) tool due to the attractiveness of its elicitation procedure and availability of software support [1], [2].

To rank a set of decision alternatives based on different criteria, a typical AHP is performed through the following four steps [3], [4].

1) Define the evaluation criteria for the decision goal and establish a hierarchical framework.

Manuscript received February 24, 2014; revised August 6, 2014; accepted September 18, 2014. Date of publication October 9, 2014; date of current version January 20, 2015. The work of P. Guan and J. Zhuang was supported in part by the United States Department of Homeland Security (DHS) through the National Center for Risk and Economic Analysis of Terrorism Events (CREATE) under Award 2010-ST-061-RE0001, and by the United States National Science Foundation under Award 1200899 and Award 1334930. Review of this manuscript was arranged by Department Editor B. Jiang. (Corresponding author: J. Zhuang.)

N. Yaraghi is with the Governance Studies, Brookings Institution, Washington, DC 20036 USA (e-mail: nyaraghi@brookings.edu).

P. Tabesh is with the Department of Management, Bauer College of Business, University of Houston, Houston, TX 77004 USA (e-mail: ptabesh@uh.edu).

P. Guan and J. Zhuang are with the Department of Industrial and System Engineering, University at Buffalo, State University of New York, New York, NY 10016 USA (e-mail: peiqiugu@ buffalo.edu; jzhuang@buffalo.edu).

Color versions of one or more of the figures in this paper are available online at http://ieeexplore.ieee.org.

Digital Object Identifier 10.1109/TEM.2014.2360082
2) Compare the pairwise decision elements.

3 ) Estimate the relative weights of decision elements.

4) Rate the decision alternatives based on aggregated weights of decision elements.

Extant literature has highlighted two limitations of the AHP methodology; first, AHP assumes that decision makers can provide precise point estimates of their preferences for alternatives, and hence, is not suitable for scenarios when there is high uncertainty in decision makers' judgment. Second, AHP does not provide any statistical interpretation of final scores of alternatives. If final scores of two alternatives are close, decision makers do not know whether there is any practical significant difference between the two alternatives.

To overcome the aforementioned limitations, many methods have been proposed, which incorporate probabilistic distributions to include uncertainty in the judgments [5]-[9]. However, in stochastic studies, enumeration of probabilities can become difficult, and hence, it is recommended to use Monte Carlo simulation approaches. Hauser and Tadikamalla [6] conclude: "For a partially or even for a completely uncertain environment, simulation is a better approach for providing a measure of confidence in a rank and for providing expected weights and ranks." Rosenbloom [7] compares the AHP and MCAHP and arrives at similar conclusions: "It should be stressed that the probabilistic interpretation is not designed to replace conventional AHP in all situations. If the decision maker feels final scores provide a clear winner there may not be a need for a probabilistic interpretation. However, if the decision maker believes the final scores are close, the probabilistic approach provides additional information."

Although the literature recommends the use of Monte Carlo analytic hierarchy process (MCAHP) in ranking alternatives under high levels of uncertainty, it does not provide clear recommendations about uncertainty levels under which the MCAHP is actually performing better than the conventional AHP. Moreover, despite the possible differences between the results of these two methods, we cannot conclude the superiority of one over another unless we have a solid basis to compare the ranking results of these two methods against that and measure the closeness of the rankings of each method to the actual rankings.

In order to overcome these issues, in this paper, we first develop and test a simulation algorithm that compares the ranking results of AHP and MCAHP methods under different levels of uncertainty. This will provide clear findings about uncertainty levels under which MCAHP outperforms the conventional AHP method. Second, we validate the simulation results by evaluating the performance of the methods in the context of blogs. We use the ranking of blogs based on preferences of 35,117 users as the 
actual ranking and measure the similarity between these rankings and the rankings acquired by AHP and MCAHP to evaluate the performance of these methods.

Analysis of the risks and uncertainties is a pivotal part of the risk management systems within organizations [10]. Our research provides empirical guidelines for risk management professionals to appropriately consider the use AHP or MCAHP in their decision making. The findings of this research would contribute to both information systems and MCDM field. To the best of our knowledge, this is the first research that evaluates AHP and MCAHP under a broad range of uncertainty levels. By comparing the performance of the two major rankings algorithms, it provides insights about the use of each method under different levels of uncertainty. The recommendations about the proper use of each method according to the level of uncertainty can be easily applied when the raters/respondents are uncertain about their judgments or when there are multiple raters/respondents with incongruent decisions. These recommendations are not limited to the application of AHP and MCAHP in the context of blog rankings and can be used in ranking a wide variety of other decision alternatives.

The rest of this paper is organized as follows: Section II discusses AHP and MCAHP and their difference in theory and practice. Section III discusses the mathematical model and shows the simulation algorithms. Section IV displays the simulation results and reveals the connections between uncertainty in input data and output rankings, as well as rank uncertainty. Section V verifies the results by applying the methods in an illustrative example of blog ranking and comparing the results of alternative methods against the actual rankings of blogs obtained through soliciting the preferences of the 35,117 users. The type and source of data are also described in the section. Finally, Section VI concludes this paper.

\section{RElated Literature}

Due to its simplicity, ease of use, and great flexibility, AHP has been applied in almost every aspect of MCDM such as project selection and evaluation, $R \& D$ ranking, and supplier selection among many others [11]-[14]. Despite the enchantment about AHP, it has also received some serious criticisms. They include the lack of a solid mathematical theory to support its axiomatic foundation [15], the shortcomings of the 1-9 scaling system in considering the uncertainty in human judgments and grasping the real human thinking system [16]-[18], its method of priority estimation [19], [20] and rank reversal phenomenon [15]. All of these criticisms have been well discussed in the literature and solutions for them have been developed. The multiattribute utility method has been used as a basis of AHP assessment methodologies [21], [22]. To take judgmental uncertainty into account, alternative methods such as applications of the fuzzy theory are developed for AHP [23], [24]. Different scaling methods have also been provided [25]-[27]. The Logarithmic leastsquares method (LLSM) was introduced as an another option to drive the weights from pairwise comparisons [28]. Crawford and Williams [28] wrote "LLSM is optimal when the judge's errors are multiplicative with a log-normal distribution. The geometric mean shares the desirable qualities of the eigenvector and is preferable in several important respects." Some other researchers have investigated different cases of rank reversal, and discussed the methods for avoiding rank reversal [29]-[31]. In many studies, the approach is to study uncertainties in imprecise judgments and probabilistic environments within stochastic or statistical context [5], [6], [32]-[34].

Saaty's AHP [1] lacks probability explanations to distinguish adjacent alternatives in final ordering. In response to this specific problem, Rosenbloom [7] suggests that, in the distribution of $1 / 9$ and 9 , where $a_{i, j}=\frac{1}{a_{i, i}}$, the pairwise values could be viewed as random variables. Therefore, it is reasonable to assume that $a_{i, j}$ is independent, and the final scores will be stochastic. Thus, instead of comparing only one single value of final scores as fixed and nonstochastic parameters, in MCAHP, the final score of an alternative is compared with others as a random variable in a prespecified confidence level. In operations research literature, Monte Carlo simulation has been combined with AHP to respond to the issue of uncertainty in judgments. Many researchers have used MCAHP, and reported the differences between its results and the results of the conventional AHP. Generally, it is expected that MCAHP produces more reliable results, which are significantly different from that of AHP [34]-[38] .

The majority of studies in the field of MCDM that adopt Monte Carlo simulation to expand the traditional AHP method follow the same theme [39]. Using an approach first proposed by Hauser and Tadikamalla [6], pairwise evaluations are randomly generated from predefined probability distributions (e.g., triangular and uniform distributions). This procedure differs from the traditional AHP in which pairwise comparisons are deterministic and are specified by the decision makers with ranges between 1 and 9 [6]. Other variations of such approach use the same basic procedure. For instance, by including different scenarios to capture the uncertainties related to future operational decisions, Levary and Wan [8] used Monte Carlo simulation to generate random numbers for scenario selection and pairwise evaluations. Other extensions to MCAHP include, but are not limited to, integrating MCAHP with approaches such as Bayesian probability [40] or adding sensitivity analysis to identify sources of variation [41].

The application of MCAHP has two advantages over the AHP method. First, it enables us to include the uncertainty of experts into the decision-making process. In reality, many decisions includes some level of uncertainty and the pairwise comparison of two decision criteria or alternatives can rarely be done without any hesitation and uncertainty. Unlike AHP, the MCAHP would allow us to define the pairwise comparisons as probability distributions rather than fixed values and thus consider the effects of uncertainties and risks in the decision-making process. The second advantages of MCAHP is that it provides a much richer output. The AHP method will only provide the rankings of the decision alternatives as fixed values, and thus, the decision maker would not know the confidence level on which the rankings are based on. When the difference in scores of the decision 
alternatives is small, this problem will be intensified. In these situations, while the difference between the alternatives may statistically be negligible and insignificant, AHP ranks them at different orders [42], [43].

This paper assumes that there are a set of different respondents that provide pairwise comparisons. We develop a model in which the importance (i.e., weights) of the decision criteria and the status of decision alternatives are simultaneously fitted to the proper probability distribution function based on the input from different pairwise comparisons of different respondents. Monte Carlo techniques are applied to simulate the performance of alternatives and weights of decision criteria as a basis of pairwise comparison. In other words, we are taking both external and internal sources of uncertainty into account. External sources of uncertainty refer to the procedure or environment for collecting preference data. In our model, we have assumed that data about decision alternatives is ambiguous. Internal sources are the ambiguity and uncertainty that result from the limited amount of information available to the decision maker and the level of his or her understanding of the problem [44]. The internal uncertainty is modeled by assuming that decision criteria weights are also ambiguous. Following Hsu and Pan [37] to obtain the probability information for $a_{i j}$ in the context of multiple decision makers, we assume that the probability of evaluations made by all respondents are equal. This will convert every $a_{i, j}$ into discrete random variables.

To the best of our knowledge, our work is the first one that compares the performance of AHP with MCAHP under a wide range of uncertainties. The current literature generally recommends MCAHP over AHP but does not clarify the extent of the superiority of the performance of MCAHP as compared with AHP. The literature does not clarify the situations under which these two methods may performs equally well. Our work bridges these gaps. We show the difference between the performances of these two methods and provide clear guidelines on how to choose the appropriate method based on the level of uncertainty in the decision problem.

\section{Simulation Algorithm}

To generate reliable data for a numerical analysis in AHP, simulation has been extensively used in prior research [45]-[48]. We also rely on simulation results to generate initial pairwise comparisons.

In the following algorithm, we generate a random but consistent matrix for the decision alternatives' performance with regard to each decision criteria as well as a random but consistent matrix to represent the weight of each decision criteria. Based on these two matrices, the overall scores and ranks of the decision alternatives are calculated. These steps are usual steps in the general AHP method. However, we try to avoid judgment bias and achieve more generalizability by creating these matrices based on pseudorandom numbers automatically generated by the computer. Steps 1 through 8 are assigned to this purpose.

We introduce uncertainty in the algorithm by creating uniform distributions based on the elements of random pairwise comparison matrices created in previous steps. In other words, instead of having a fixed parameter, we have a random variable with a uniform distribution as the pairwise comparison of two alternatives. As we slightly increase the range of the uniform distribution, the uncertainty increases as well. We calculate the final scores and ranks for 1000 different iterations at each level of uncertainty and compare them with the scores and ranks that we first created. The algorithm is formally described in the 12 steps and graphically shown in Fig. 1.

Step 1: Suppose we have $N$ different alternatives and $M$ different decision criteria. We create a $N \times N$ random matrices $A^{m}, m=1,2, \ldots, M$, to represent the comparison of $N$ alternatives with regards to the $m$ th decision criterion

$$
A^{m}=\left[\begin{array}{cccc}
a_{1,1}^{m} & a_{1,2}^{m} & \cdots & a_{i, n}^{m} \\
a_{2,1}^{m} & a_{2,2}^{m} & \cdots & a_{2, n}^{m} \\
\vdots & \vdots & \ddots & \vdots \\
a_{n, 1}^{m} & a_{n, 2}^{m} & \cdots & a_{n, n}^{m}
\end{array}\right], \quad m=1,2, \ldots, M .
$$

The elements in the upper triangular section of the matrix are shown by $a_{i, j}^{m}$, which is a random variable and follows a uniform distribution in the domain of $[1,9] \forall i=1,2, \ldots, n ; i<j$. The elements in the lower triangular section of the matrix $a_{j, i}^{m}$ are defined as $a_{i, j}^{m}=\frac{1}{a_{j, i}^{m}}$. In the matrix $A^{m}, a_{i, j}^{m}$ represents the score of decision alternative $i$ as compared with alternative $j$ with regard to decision criterion $m$ in a linear scale.

Although prior research has proposed many alternative scales such as power scale and root square [49], geometric [25], logarithmic [50], asymptotical [51], inverse linear [52], and balanced [53], we limit our simulation to the most widely used linear scales to avoid further complications in the algorithm and simulation process.

Step 2: For each matrix, we normalize each element by the column sum

$$
\dot{a}_{i, j}^{m}=\frac{a_{i, j}^{m}}{\sum_{i=1}^{n} a_{i, j}^{m}}, \text { for } m=1,2, \ldots, M .
$$

Step 3: We calculate the geometric mean of each row to have a $n \times 1$ matrix

$$
\omega^{m}=\left[\begin{array}{c}
\left(\prod_{i=1}^{n} \dot{a}_{1, i}^{m}\right)^{1 / n} \\
\left(\prod_{i=1}^{n} \dot{a}_{2, i}^{m}\right)^{1 / n} \\
\vdots \\
\left(\prod_{i=1}^{n} \dot{a}_{n, i}^{m}\right)^{1 / n}
\end{array}\right], \text { for } m=1,2, \ldots, M .
$$

This matrix shows the estimated weights of each decision alternative regarding a single decision criterion.

Step 4: We check for consistency in each of the matrices that we have randomly created. If the consistency ratio of the matrices is less than 0.1 , then we let $m=m+1$. 


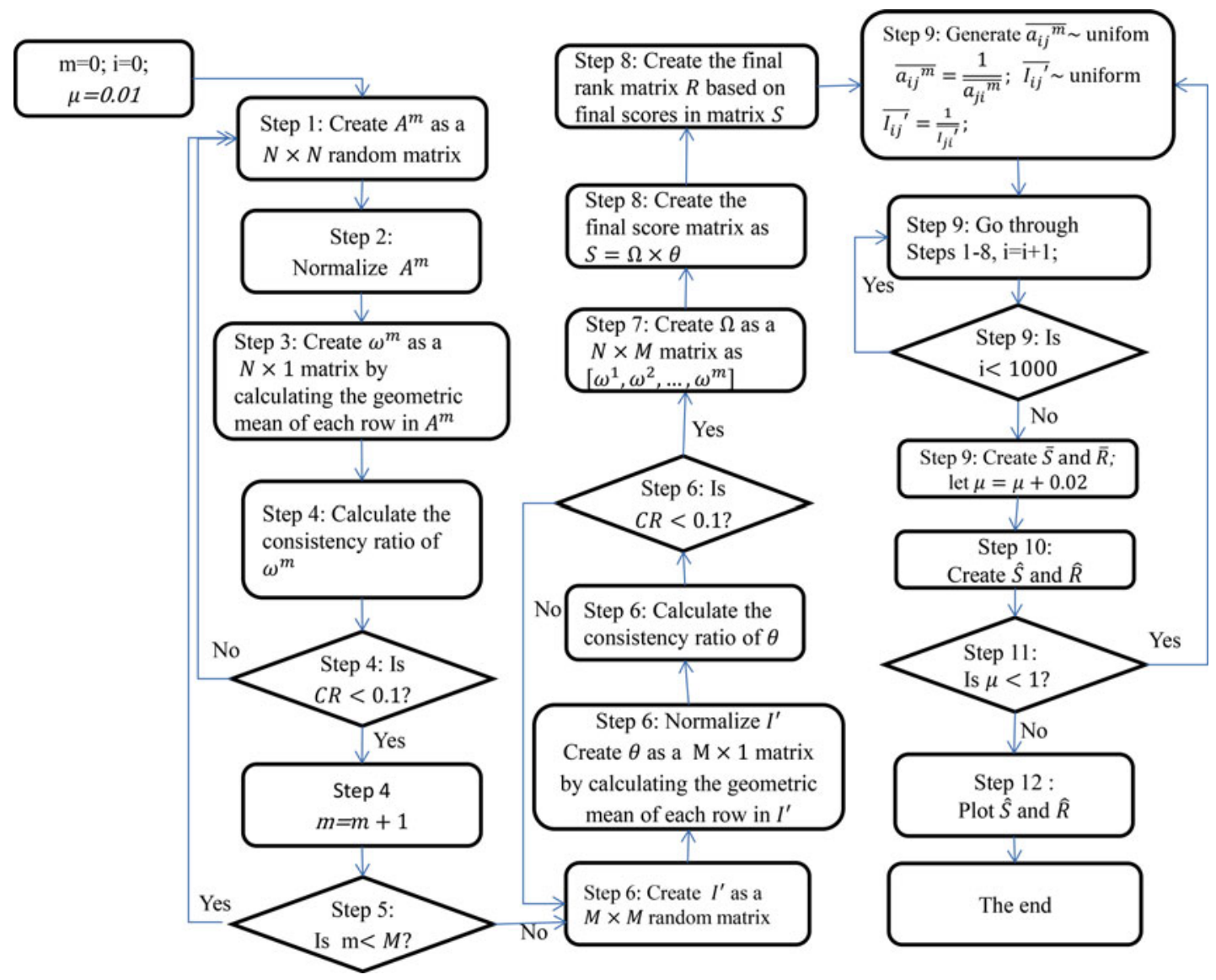

Fig. 1. Graphical representation of simulation algorithm

Step 5: Continue to create random and consistent $A^{m}$ matrices through Steps 1-4 until $M$ consistent $A^{m}$ matrices are available. If the random matrices generated before have a consistency ratio higher than 0.1 , the algorithm returns to Step 1 .

Step 6: We create $I^{\prime}$ as a $M \times M$ random matrix just like the previous matrices to represent the weights of the decision criteria with regard to each other. We follow the previous steps $(2,3$, and 4$)$ to be sure that the matrix is consistent, and thus, have the final $M \times 1$ matrix called $\theta$.

Step 7: We create the $\Omega$ matrix as $\left[\omega^{1}, \omega^{2}, \ldots, \omega^{M}\right]$, which is a $N \times M$ matrix.

Step 8: We multiply $\Omega$ by $\theta$ to find a $N \times 1$ matrix, which shows the final score of each decision alternative based on all of the factors. Matrix $S$ is calculated as $S=\Omega \times \theta$. Based on the scores in matrix $S$, we calculate the ranks of each alternative in matrix $R$. Now we have our initial set of matrices, and the scores and ranks of alternatives based on these random but consistent matrices.

Step 9: We proceed to generate a random matrix in correspondence with each of the $A^{m}$ and $I^{\prime}$ matrices such as

$$
\begin{aligned}
& \overline{a_{i, j}^{m}} \sim \text { Uniform }\left[\max \left(0, a_{i, j}^{m}-\mu a_{i, j}^{m}\right), \min \left(9, a_{i, j}^{m}+\mu a_{i, j}^{m}\right)\right] \\
& \text { and } \overline{a_{i, j}^{m}}=\frac{1}{\overline{a_{j, i}^{m}}}
\end{aligned}
$$

$\overline{I_{i, j}^{\prime}} \sim$ Uniform $\left[\max \left(0, I_{i, j}^{\prime}-\mu I_{i, j}^{\prime}\right), \min \left(9, I_{i, j}^{\prime}+\mu I_{i, j}^{\prime}\right)\right]$

$$
\text { and } \overline{I_{j, i}^{\prime}}=\frac{1}{\overline{I_{j, i}^{\prime}}}
$$

The bar notation in $\overline{a_{i, j}^{m}}$ is used to differentiate the elements of the new matrix from the initial matrix $A^{m}$, and at the same time, indicates that the elements of the new matrix, $\overline{A^{m}}$ are created based on the initial values of the previous matrix, $A^{m}$. In other words, $\overline{a_{i, j}^{m}}$ s are created as random draws form uniform distributions, which are centered on $a_{i, j}^{m}$ s. As shown in (6) and (7), to consider the uncertainty in the weights of the decision criteria, we create the new matrix $\overline{I^{\prime}}$ in a similar way.

The parameter $\mu$ indicates the level of uncertainty in the simulation algorithm and is initially set at 0.01 . At this step, we generate 1000 random draws for each $\overline{a_{i, j}^{m}}$ and $\overline{I_{j, i}^{\prime}}$ from the uniform distribution defined in (4)-(7). In each of these 1000 iterations, we go over Steps 1 to 8 in order to create the consistent matrices, and calculate the scores and the ranks of the alternatives. We will have 1000 different scores and ranks for each of the decision alternatives. To summarize these 1000 scores and ranks into a single value, we will consider $\bar{S}$ as their average score and $\bar{R}$ as their corresponding rank to, respectively, represent the final score and rank of the alternatives at each particular level of uncertainty.

To allow for different levels of uncertainty, we gradually increase $\mu$ from 0.01 to 1 in 0.02 increment. At each increment, a new average score and average rank $(\bar{S}$ and $\bar{R})$ is calculated as discussed previously. The incremental increase in the value 


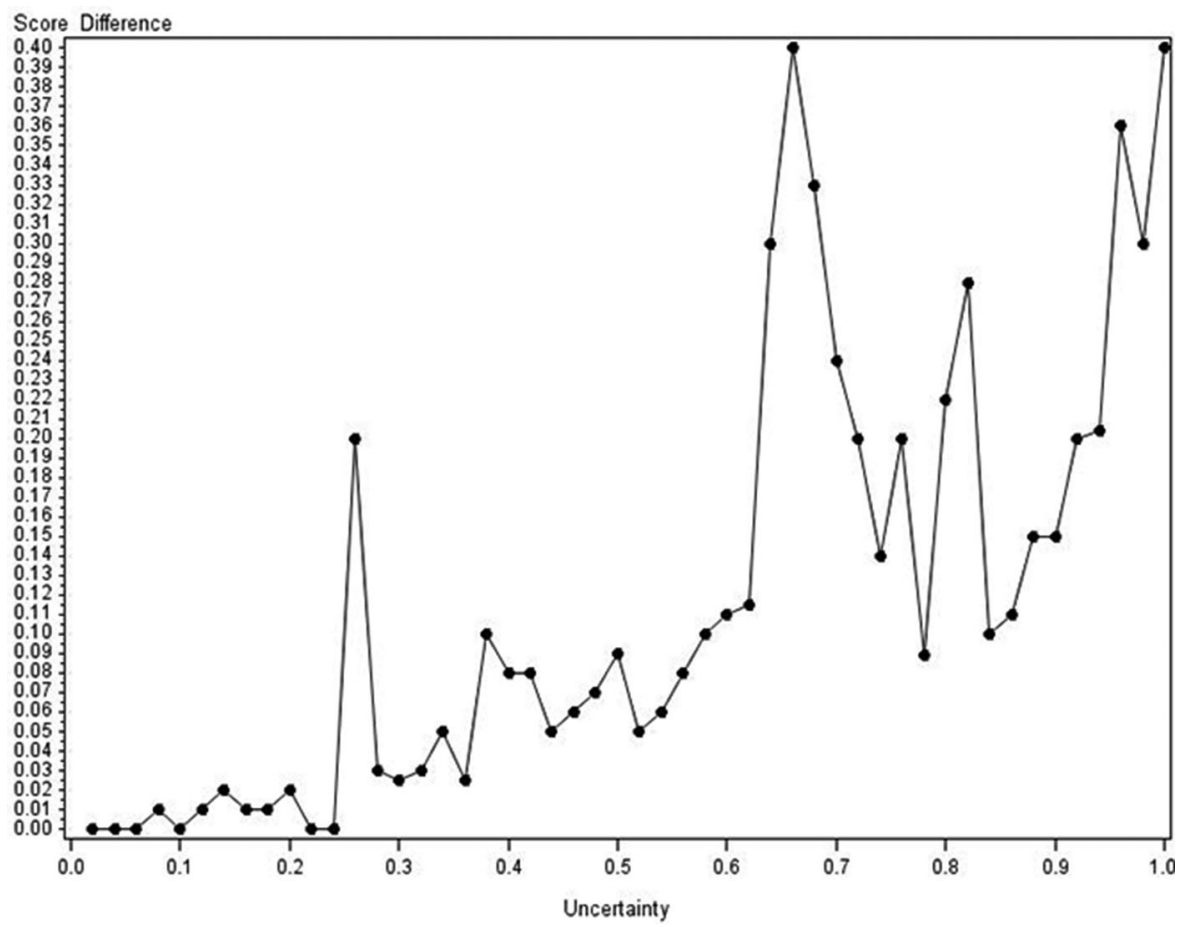

Fig. 2. Difference between scores in Monte Carlo AHP and AHP.

of $\mu$ allows us to investigate the differences between the performance of the two methods over a wide range of uncertainties. This implies that the uncertainty in the model is reflected by $\mu$ and is proportionate to the coefficient of variation in a uniform distribution of pairwise comparisons.

The degree of uncertainty in the simulation algorithm is gauged based on the range of the corresponding uniform distributions defined in (4) and (6). We assume that as the lower and upper bounds of the uniform distribution from which we draw our random values increase, the variation in the values of the random values also increases. This would imply that as the range of the uniform distribution increases, the level of uncertainty in the decision-making process also increases. Since we did not have any theory in the literature to help us assume a prespecified level of uncertainty in our study, we design our simulation algorithm in such a way that a wide spectrum of possible uncertainty levels can be taken into account. That is, levels of the uncertainty from 0.01 to 1.00 are simulated in 0.02 increments. Both the 0.01-1 range and the 0.02 increments are designed to accommodate the highest uncertainty levels that we could simulated given our computational resources.

Let $\mu=\mu+0.02$.

Step 10: At each uncertainty level of $\mu$, we calculate the difference between the initial scores and the ranks with $\bar{S}$ and $\bar{R}$

$$
\hat{S}=\sqrt{\left(\overline{S_{n}}-S_{n}\right)^{2}}, \hat{R}=\sqrt{\left(\overline{R_{n}}-R_{n}\right)^{2}} .
$$

In which, $n=1,2, \ldots, N$. In these equations, $S_{n}$ and $R_{n}$ represent the score and rank of the $n$th alternative, respectively, obtained from the AHP method and $\overline{S_{n}}$ and $\overline{R_{n}}$ represent the score and ranks of $n$th alternative, respectively, obtained from the Monte Carlo AHP method.

Note that $\hat{S}$ and $\hat{R}$, respectively, measure the difference in scores and ranks of decision alternatives under two scenarios. In the first scenario, there is no uncertainty in the decision process. $S$ and $R$ are calculated under this scenario. In the second scenario, some level of uncertainty in introduced in the decision process by inclusion of $\mu$ as discussed in step 9. The final scores and ranks of the decision alternatives in the second scenario are captured by $\bar{S}$ and $\bar{R}$.

Step 11: If $\mu<1$, we return step 9 and recalculate $\hat{S}$ and $\hat{R}$ for each value of $\mu$.

Step 12: We plot the values of $\hat{S}$ and $\hat{R}$ over the change of $\mu$. The end.

\section{Simulation Results}

In this section, we present the results of our simulation study. Note that the purpose of the simulation algorithm was to compare the performance of AHP with the MCAHP method in terms of score and ranks of decision alternatives for a different levels of uncertainty ranging from 0.01 to 1 . The summary of the simulation results are presented in Figs. 2 and 3.

We run the code for five decision alternatives and five decision criteria. There are 1000 iterations in each level of the uncertainty where there are 50 different levels of uncertainty ranging from 0.01 to 1 . In Fig. 2, we show the difference between scores of the MCAHP and the conventional AHP. On the horizontal axis, the 50 different levels of uncertainty are shown and the difference between the scores in two methods is plotted against the vertical axis. Despite the random peaks, difference between the scores 


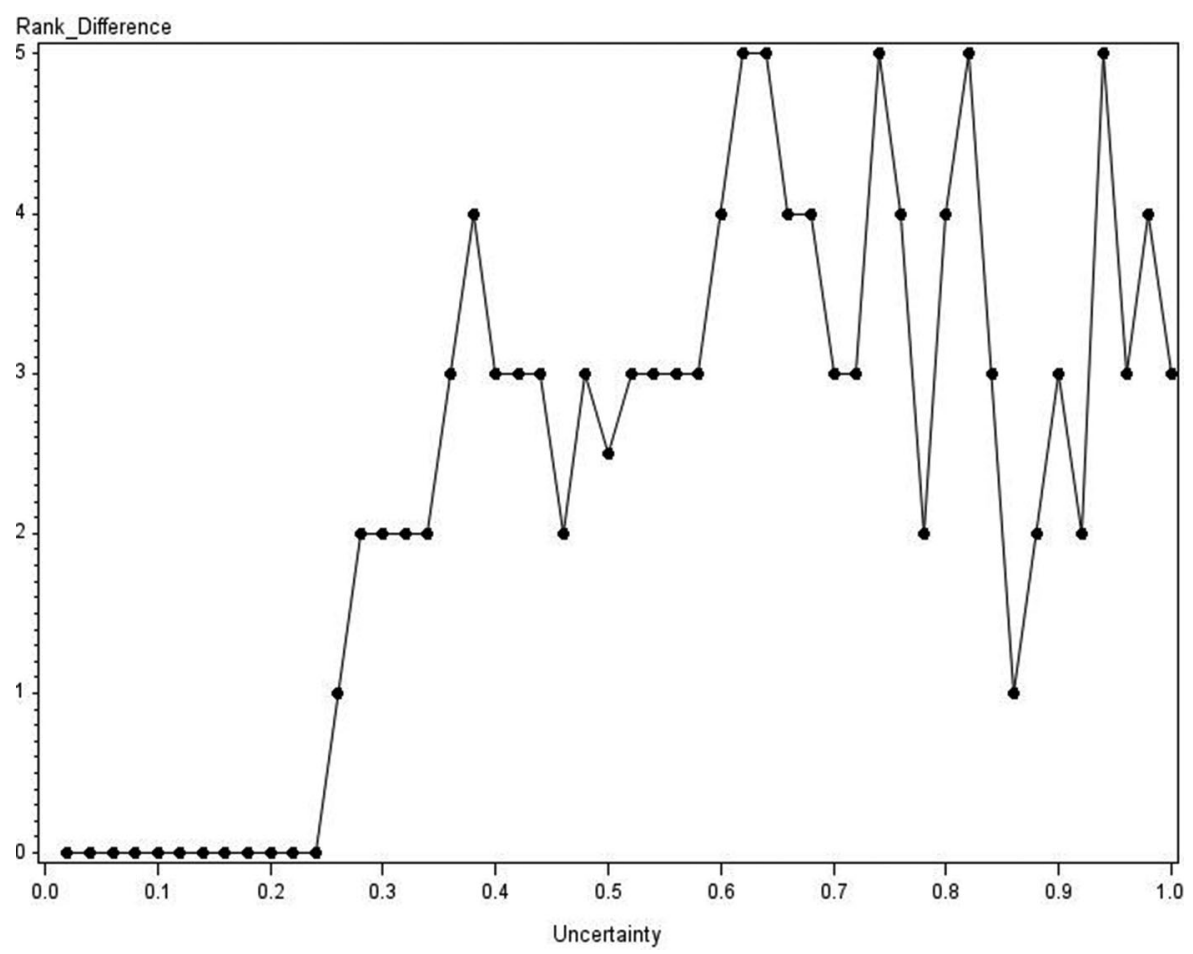

Fig. 3. Difference between ranks in Monte Carlo AHP and AHP

calculated based on the two different methods increases as the level of the uncertainty increases. However, one interesting observation is that difference between the scores is insignificant as long as the level of uncertainty is lower than 0.24 . Fig. 3 shows the differences between the final ranks, which are calculated based on the final scores, and as expected, the difference between the ranks is zero until the uncertainty level of 0.24 . It is interesting to note that despite the increases in the uncertainty level, the final scores do not dramatically change in the higher levels.

The simulation results reveal an interesting point about the situations in which there is a significant difference between MCAHP and AHP results. Based on these results, as long as the uncertainty measure is less than 0.24 , both methods produce similar results. To further investigate the difference between the scores and ranks in two methods, we use a one-sample $t$-test and investigate the null hypothesis that the difference between the values in each uncertainty level is equal to zero. Fig. 4 shows the respective $p$-values for each uncertainty level. The $p$-values corresponding to uncertainty levels lower than 0.24 are all above 0.05 , and thus, the hypothesis that the methods produce equal ranks and scores are not rejected. However, after the 0.24 level of uncertainty $(\mu>0.24)$, our statistical test confirms the visual conclusions we drew before.

Our simulation results considers only two levels of hierarchy and includes the ranking of five alternatives with regards to five criteria. The other assumption of our model is the independency of decision criteria from each other. This assumption has been enforced in the simulation algorithm by sampling the pairwise comparisons as independent random variables from a uniform distribution. The limitations of these assumptions are discussed at Section VI in further details.
In summary, our simulation results show that the performance of MCAHP is not significantly different from AHP as long as the level of uncertainty is below 0.24 . As the uncertainty increases, the two methods provide different results. Although our simulation results identify the threshold on which MCAHP results are different from AHP, we still have to investigate if the MCAHP results are better than AHP. To do so, we need to compare each set of rankings provided by AHP and MCAHP methods with a ranking that has been already produced by an alternative, yet reliable ranking method. This alternative ranking will be considered as a basis, or actual ranking of the alternatives and will be used to measure the closeness of the rankings provided by AHP and MCAHP to reality. These evaluations are provided in Section V.

\section{APPLiCATION AND EVALUation OF AHP AND MCAHP IN RANKING BLOGS}

In this section, we measure the closeness of the ranking results of the AHP and MCAHP to other widely used ranking systems. Blogs constitute a suitable context for this purpose since they have been already ranked by many reliable ranking systems other than AHP or MCAHP. The recommendations of this paper would be applicable in any context. Here, we use blog (or weblog) rankings as an illustrative example for two reasons; first, the subject is of interest to many information systems researchers and second, as mentioned before, an alternative ranking of blogs is already available. This alternative ranking is based on the recommendations of a very large user base and can be considered as a solid ranking. We used this ranking to evaluate the performance of both MCAHP and AHP methods 


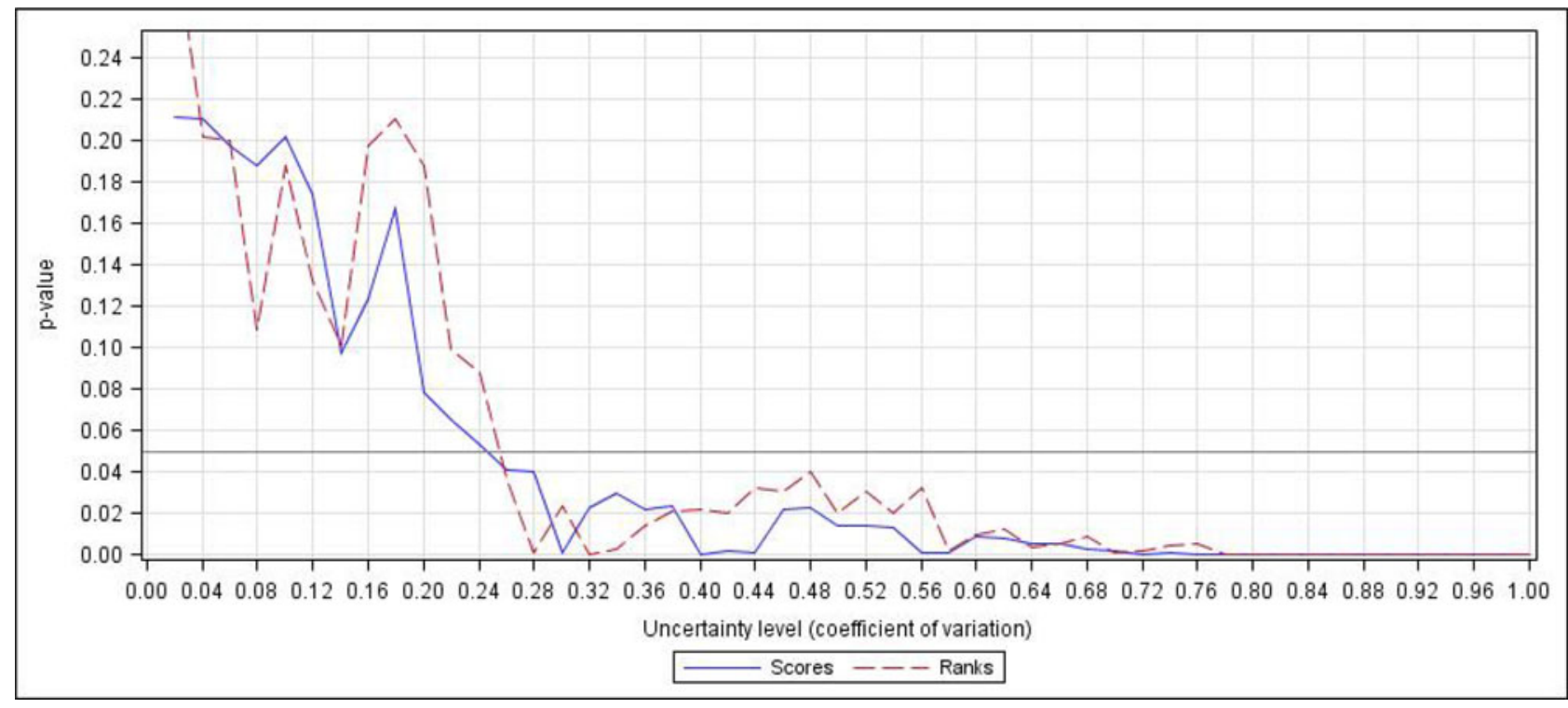

Fig. 4. Corresponding $p$-values of testing null hypothesis that the results of the two methods are equal. Blue line reflects the $p$-values of testing the equality of the scores in two methods. Red line reflects the $p$-values of testing the equality of the ranks in two methods.

against it. We evaluate the results of AHP and MCAHP with the alternative rankings in two situations. The first situation only considers the blogs, which are very close to each other in terms of overall quality. This implies that it would be more difficult for the respondents to perform a pairwise comparison on these blogs, and thus, their evaluations would entail a high degree of uncertainty. The second situation only considers the blogs that are very different in terms of their overall quality, and thus, can beyes ranked much easier and with a lower degree of uncertainty. The details of the alternative ranking method discussed later.

Until its discontinuation on July 1st, 2013, readership and subscription to blogs was facilitated through a service by Google called Reader [54]. Users could subscribe, share, comment, and also give positive feedback by clicking a button called "like" for each post of the blogs they read. Using Google application programming interfaces, Likekhor.com [55] gathered all of this information about 2654 blogs in Persian blogosphere and provided different rankings of the blogs based on the information from 35117 users. There were four ranking systems, based on the following: 1) the number of subscribers; 2) the total number of "likes;" 3 ) the average "likes" for each post; and 4) a combination of all three measures. We have collected the data on the rankings of the blogs in January 2013. The last ranking system (number 4) provides the most reliable rankings because it entails aspects of popularity, quality, and readership of the blog. Since the rankings are based on the votes by a fairly large population of users, we consider the fourth ranking system as the actual (real) or at least the best possible ranking of the blogs.

Ten different blogs were chosen as decision alternatives, five of which are fairly close to each other (no more than two places of difference in the ranking), and the other five, are those with larger gaps between them (more than five places of difference between them). We only chose the blogs that publish posts in economics and management issues. We chose these blogs to filter prejudices and conflicts that may results among raters due to different points of view in sociocultural issues. Two groups of blogs are presented to ten raters. Raters were chosen among Persian MBA students in University at Buffalo and the University of Houston to ensure the familiarity of the raters with the topics of the blogs. The first five blogs in group 1, represent choices with high uncertainty since they are actually very close to each other and differentiating among them is harder for readers, where the second group of blogs with wider gaps of rankings represent the alternatives with a lower degree of uncertainty since the difference among them is more significant and easier to distinguish. We rely on the study of Kaye and Johnson [56] to define the factors associated with blog credibility from the view point of readers. They identify ten different reasons that readers read blogs and judge their credibility. Some of these factors are highly related to each other, and will not provide additional information when used together in ranking algorithms. Based on these ten reasons, we identified the following four factors as our decision criteria.

1) The information on the blog is not available through main stream media.

2) The blog provides useful links to additional information source.

3) The blog provides up-to-date and relevant information.

4) The blog provides interesting analysis of current events.

"Decision criteria" refers to the elements based on which different decision options are evaluated. In our context, the above four factors are considered as the decision criteria for ranking blogs. "Pairwise comparison" refers to the evaluation of each of the two decision options or criteria. In AHP and MCAHP methods, the pairwise comparison is used to calculate the relative importance of each decision criterion. Each pair of available options are also evaluated against each other by pairwise 
TABLE I

ACTUAL, GROUP, AND BEST INDIVIDUAL RANKINGS

\begin{tabular}{|c|c|c|c|c|c|}
\hline $\begin{array}{l}\text { Level of } \\
\text { uncertainty }\end{array}$ & Alternatives & $\begin{array}{l}\text { Actual ratings (Based } \\
\text { on Likekhor.com) among } \\
\text { all the existing blogs }\end{array}$ & $\begin{array}{l}\text { Rankings among five } \\
\text { alternatives based on } \\
\text { actual rankings }\end{array}$ & $\begin{array}{c}\text { Group rankings } \\
\text { (Based on the average } \\
\text { of the group) }\end{array}$ & $\begin{array}{l}\text { Best } \\
\text { individual } \\
\text { ranking }\end{array}$ \\
\hline \multirow[t]{5}{*}{ High } & $\mathrm{B} \log \mathrm{A}$ & 4 & 1 & 2 & 1 \\
\hline & Blog B & 5 & 2 & 3 & 3 \\
\hline & $\mathrm{B} \log \mathrm{C}$ & 6 & 3 & 1 & 4 \\
\hline & $\mathrm{B} \log \mathrm{D}$ & 8 & 4 & 5 & 5 \\
\hline & $\mathrm{B} \log \mathrm{E}$ & 10 & 5 & 4 & 2 \\
\hline \multirow[t]{5}{*}{ Low } & $\mathrm{B} \log \mathrm{F}$ & 1 & 1 & 1 & 2 \\
\hline & $B \log G$ & 14 & 2 & 3 & 3 \\
\hline & $\mathrm{B} \log \mathrm{H}$ & 25 & 3 & 4 & 1 \\
\hline & Blog I & 40 & 4 & 2 & 4 \\
\hline & Blog J & 46 & 5 & 5 & 5 \\
\hline
\end{tabular}

comparison. In the setting of blog rankings, the four decision criteria are evaluated against each other in pairs, that is criterion 1 with 2 , criterion 1 with 3 , and so on. This results in a $4 \times 4$ matrix in which the element in each cell represents the relative importance of one criterion compared to the other. For example, if a rater believes that the criterion 1: "The information on the blog is not available through main stream media" is twice as important as the criterion 2: "The blog provides useful links to additional information source" then the element on row 1 , column 2 of the matrix would be 2 . The "decision alternatives" refers to the available options that should be ranked and the "status of the decision alternatives" refers to the relative rank of the of the decision alternatives based on the pairwise evaluation of respondents about the performance of the alternatives in each decision criterion.

Each rater was briefed about the AHP method, and then, was asked to do pairwise comparisons for two different sets of five blogs. The consistency index for each of the pairwise comparisons was checked, and none was higher than 0.07, and thus, the comparisons were all consistent. With ten separate sets of pairwise comparisons for each of the two categories, we construct two ranking systems: 1) based on the unweighted average of the final rankings as the group decision and 2) based on the most similar ranking (the one with the lowest square difference in rankings) as the best individual ranking. The results are shown in Table 1 for each of the two groups of blogs; the first five rows reflect the blogs that belong to the high uncertainty group and the last five rows show the blogs in the low uncertainty group.

Note that in Table I, the first five alternatives are those with the lowest difference in actual rankings; and as we discussed earlier in this section, those are considered the ones with the highest degree of uncertainty. The alternatives listed in the five lower rows of the table, are those with the largest difference in actual rankings, and thus, are considered the ones with the lower uncertainty level. The last two columns show the rankings based on the first two ranking systems: the first ranking system is based on the average of ten judgments, and the last one is the best individual judgments, which is the nearest ranking to the actual ranking. Table II reveals interesting findings about the two methods in two different scenarios. In each cell, the sum of
TABLE II

COMPARISON OF GROUP AND BEST INDIVIDUAL RANKINGS

\begin{tabular}{lcc}
\hline \hline & $\begin{array}{c}\text { Group } \\
\text { decision }\end{array}$ & $\begin{array}{c}\text { Best individual } \\
\text { decision }\end{array}$ \\
\hline Alternatives with high uncertainty & 8 & 12 \\
Alternatives with low uncertainty & 6 & 6 \\
\hline \hline
\end{tabular}

square differences between the rankings and the actual rankings are presented. When the level of uncertainty increases, the group makes better decisions as compared with the best individual in the group. On the other hand, when the level of uncertainty is low, the group and the best individual judgments are not different from each other.

We further use the collected data about the pairwise comparison as the initial input data for the MCAHP according to the following steps.

We use the comparison datasets to model the probability distribution function (pdf) of each of the elements of pairwise comparison weights' matrix by the following four steps.

1) Estimate parameter values of each distribution by the maximum-likelihood estimators (MLEs of each distribution).

2) Optimize the fit using the Levenberg-Marquardt method.

3) Measure the goodness-of-fit for each of the optimized functions using chi-square, Kolmogorov-Smirnov and/or Anderson-Darling tests.

4) Select the best fit by comparing all functions and choosing the one with the lowest goodness-of-fit values. The pdfs were then used to simulate 1000 iterations of pairwise comparisons.

Table III shows the MCAHP results in the two different scenarios as compared with the actual ratings. For each of the two groups of blogs; the first five rows reflect the blogs that belong to the high uncertainty group and the last five rows show the blogs in the low uncertainty group.

The sum of the square difference between the results of the MCAHP rankings in the scenario of low uncertainty is 6 , which is the same as the other two methods; however, in the case of high uncertainty, the sum of the square of differences between 
TABLE III

COMPARISON OF ACTUAL (LiKEKHOR.COM) AND MONTE CARLO AHP RANKINGS

\begin{tabular}{lcccc}
\hline \hline $\begin{array}{l}\text { Level of } \\
\text { uncertainty }\end{array}$ & Alternatives & $\begin{array}{c}\text { Actual ratings (based } \\
\text { on Likekhor.com) among } \\
\text { all the existing blogs }\end{array}$ & $\begin{array}{c}\text { Rankings among five } \\
\text { alternatives based on } \\
\text { actual rankings }\end{array}$ & $\begin{array}{c}\text { Monte Carlo } \\
\text { AHP } \\
\text { rankings }\end{array}$ \\
\hline High & Blog A & 4 & 1 & 1 \\
& Blog B & 5 & 2 & 2 \\
& Blog C & 6 & 3 & 3 \\
Low & Blog D & 8 & 4 & 5 \\
& Blog E & 10 & 5 & 4 \\
& Blog F & 1 & 1 & 1 \\
Blog G & 14 & 3 & 3 \\
Blog H & 25 & 4 & 4 \\
\hline \hline & Blog I & 40 & 5 & 5 \\
\hline
\end{tabular}

the MCAHP and the actual rankings is only 2 . These results partially confirm the previous research findings, which state that the MCAHP performs better than AHP; however when the uncertainty is not substantially high, the results do not differ from the other two methods.

\section{CONCLUSION}

In this paper, we use simulation results to compare the performance of MCAHP with the AHP method. Although it is generally accepted that the first method performs better than AHP, it has not been clear that how the performance changes in accordance with the level of uncertainty. This paper shows that the difference between the results is not significant when the level of uncertainty is less than 0.24 . Although this paper addresses one of the most important limitations in the literature regarding the comparison of the two methods based on both simulated and real data, it can be extended by considering higher levels of uncertainty. Moreover, we have validated our findings by a real-world example of Persian blogs. This practical example not only validates our results, but also is of interest to managerial decision-making researchers as a new method for selecting the proper tool for ranking among alternatives.

Our simulation results considers only two levels of hierarchy and includes the ranking of five alternatives with regards to five criteria; the hierarchy in the practical example is also structured in two levels, however, it has four evaluation criteria. Increasing the levels of hierarchy and the number of criteria and alternatives would affect the performance of AHP and MCAHP, and shedding light on these effects warrants further simulation studies on different formats of hierarchies with different numbers of criteria and alternatives. However, as mentioned in the beginning of Section IV our simulation algorithm demands a huge level of computation power. Increasing the levels of hierarchy, the number of decision criteria or alternatives would increase the computing time exponentially, and would not result in satisfactory outputs in a reasonable time window. We believe that further investigation on the effects of these complexities on the performance of these methods should be undertaken. We are now working on optimizing our simulation algorithm to reduce the computation time. This will enable us to further in- vestigate more complicated hierarchies with higher number of decision alternatives and criteria.

Although higher levels of uncertainty can be simulated with more powerful computers, it would not add to our results since we have already shown that in uncertainty levels higher that 0.24, MCAHP would perform better that AHP. More detailed simulations can be performed to analyze the performance of the two methods by increasing the uncertainty levels in increments smaller than 0.02. In this study, we show the statistical difference between the performance of AHP and MCAHP does not exist at uncertainty level of 0.22 , but appears at 0.24 . The performance of the methods between these two levels of uncertainty is unclear in our study. More power computer technology and a better algorithm design would enable us to study the performance of the methods in smaller algorithms, and thus, understand how these two methods perform within the range of 0.22 and 0.24 .

Another limitation of our study that should be addressed by future research is the assumption of independence among valuecreating criteria in traditional MCDM method. In real-world problems, decision-making criteria may be directly or indirectly interrelated [57]. Moreover, alternatives may be evaluated against aspiration levels (e.g., goals) rather than relative to other existing alternatives in the vicinity of the problem [58]. Therefore, statistical and economic assumptions might be at times unrealistic, and thus, creating new problems rendering the traditional MCDM methods ineffective [57], [59] . These important issues have been theorized and addressed in the recent MCDM literature by introducing hybrid multicriteria models [57], [59], [60]. For instance, using tools such as decision-making trial and evaluation laboratory (DEMATEL) is suggested by Tzeng $\mathrm{et} \mathrm{al}$. [57] to build a relation structure among the criteria (see Liou [61] for detailed explanations for improved MCDM approaches).

Our methodological comparisons are not affected by this issue since our assumptions are clear and our method specifically uses random independent variables for generating the relative score of a decision alternative with regard to a specific decision criterion. Therefore, the independence of the criteria is assumed and properly enforced in the simulation algorithm. Hence, the simulation results and the major contribution of the study to compare two MCDM methods are not being affected by unspecified issues. However, in the real-world application of our 
method in blog rankings, criteria may be mutually interrelated. To maintain parsimony and focus on main contribution of our study (comparison of AHP and MCAHP under different levels of uncertainty), we assume independence of criteria in our illustrative example of blog ranking as well. Further implications of using techniques such as DEMATEL to increase the effectiveness of AHP and MCAHP need to be addressed by future research.

\section{REFERENCES}

[1] T. L. Saaty, The Analytic Hierarchy Process: Planning, Priority Setting, Resources Allocation. New York, NY, USA: McGraw-Hill, 1980.

[2] R. P. Hämäläinen, "Reversing the perspective on the applications of decision analysis," Decision Anal., vol. 1, no. 1, pp. 26-31, 2004.

[3] F. Zahedi, "The analytic hierarchy process-a survey of the method and its applications," Interfaces, vol. 16, no. 4, pp. 96-108, 1986.

[4] T. L. Saaty, "How to make a decision: The analytic hierarchy process," Eur. J. Oper. Res., vol. 48, no. 1, pp. 9-26, 1990.

[5] D. Paulson and S. Zahir, "Consequences of uncertainty in the analytic hierarchy process: A simulation approach," Eur. J. Oper. Res., vol. 87, no. 1, pp. 45-56, 1995.

[6] D. Hauser and P. Tadikamalla, "The analytic hierarchy process in an uncertain environment: A simulation approach," Eur. J. Oper. Res., vol. 91, no. 1, pp. 27-37, 1996.

[7] E. S. Rosenbloom, "A probabilistic interpretation of the final rankings in AHP,” Eur. J. Oper. Res., vol. 96, no. 2, pp. 371-378, 1997.

[8] R. R. Levary and K. Wan, "A simulation approach for handling uncertainty in the analytic hierarchy process," Eur. J. Oper. Res., vol. 106, no. 1, pp. 116-122, 1998.

[9] R. Bañuelas and J. Antony, "Application of stochastic analytic hierarchy process within a domestic appliance manufacturer," J. Oper. Res. Soc., vol. 58 , no. 1 , pp. 29-38, 2006

[10] N. Yaraghi and R. G. Langhe, "Critical success factors for risk management systems," J. Risk Res., vol. 14, no. 5, pp. 551-581, 2011

[11] E. K. Lee and S. K. Ha, S.and Kim, "Supplier selection and management system considering relationships in supply chain management," IEEE Trans. Eng. Manage., vol. 48, no. 3, pp. 307-318, Aug. 2001.

[12] O. S. Vaidya and S. Kumar, "Analytic hierarchy process: An overview of applications," Eur. J. Oper. Res., vol. 169, no. 1, pp. 1-29, 2006.

[13] W. R. Bitman and N. Sharif, "A conceptual framework for ranking R\& D projects," IEEE Trans. Eng. Manage., vol. 55, no. 2, pp. 267-278, May. 2008.

[14] W. Ho, "Integrated analytic hierarchy process and its applications-A literature review," Eur. J. Oper. Res., vol. 186, no. 1, pp. 211-228, 2008.

[15] J. S. Dyer, "Remarks on the analytic hierarchy process," Manage. Sci., vol. 36, no. 3, pp. 249-258, 1990.

[16] S. R. Watson and A. N. S. Freeling, "Comment on: Assessing attribute weights by ratios," Omega, vol. 11, no. 1, p. 13, 1983.

[17] A. Arbel, "Approximate articulation of preference and priority derivation," Eur. J. Oper. Res., vol. 43, no. 3, pp. 317-326, 1989.

[18] B. Schoner and W. C. Wedley, "Ambiguous criteria weights in AHP: Consequences and solutions," Decision Sci., vol. 20, no. 3, pp. 462-475, 1989.

[19] C. A. B. Costa and J. C. Vansnick, "MACBETH - An interactive path towards the construction of cardinal value functions," Int. Trans. Oper. Res., vol. 1, no. 4, pp. 489-500, 1994.

[20] C. A. B. Costa and J. C. Vansnick, "A critical analysis of the eigenvalue method used to derive priorities in AHP," Eur. J. Oper. Res., vol. 187, no. 3, pp. 1422-1428, 2008.

[21] P. T. Harker and L. G. Vargas, "Reply to "remarks on the analytic hierarchy process" by JS Dyer," Manage. Sci., vol. 36, no. 3, pp. 269-273, 1990.

[22] R. Ramanathan, "A note on the use of the analytic hierarchy process for environmental impact assessment," J. Environ. Manage., vol. 63, no. 1, pp. 27-35, 2001.

[23] D. Y. Chang, "Applications of the extent analysis method on fuzzy AHP," Eur. J. Oper. Res., vol. 95, no. 3, pp. 649-655, 1996.

[24] L. C. Leung and D. Cao, "On consistency and ranking of alternatives in fuzzy AHP," Eur. J. Oper. Res., vol. 124, no. 1, pp. 102-113, 2000.

[25] F. A. Lootsma, "Conflict resolution via pairwise comparison of concessions," Eur. J. Oper. Res., vol. 40, no. 1, pp. 109-116, 1989.
[26] F. A. Lootsma, "Scale sensitivity in the multiplicative AHP and SMART," J. Multi-Criteria Decision Anal., vol. 2, no. 2, pp. 87-110, 1993.

[27] Y. Dong, G. Zhang, W. Hong, and Y. Xu, "Consensus models for AHP group decision making under row geometric mean prioritization method," Decision Support Syst., vol. 49, no. 3, pp. 281-289, 2010.

[28] G. Crawford and C. Williams, "A note on the analysis of subjective judgment matrices," J. Math. Psychol., vol. 29, no. 4, pp. 387-405, 1985.

[29] J. Barzilai and B. Golany, "AHP rank reversal, normalization and aggregation rules," Inf. Syst. Oper. Res., vol. 32, no. 2, pp. 57-64, 1994.

[30] S. Schenkerman, "Avoiding rank reversal in AHP decision-support mode," Eur. J. Oper. Res., vol. 74, no. 3, pp. 655-688, 1994.

[31] A. Stam and A. Silva, "Stochastic judgments in the AHP: The measurement of rank reversal probabilities," Decision Sci., vol. 28, no. 3, pp. 655-688, 1997.

[32] M. Sajjad Zahir, "Incorporating the uncertainty of decision judgements in the analytic hierarchy process," Eur. J. Oper. Res., vol. 53, no. 2 , pp. 206-216, 1991.

[33] I. Basak and T. Saaty, "Group decision making using the analytic hierarchy process," Math. Comput. Modelling, vol. 17, no. 4, pp. 101-109, 1993.

[34] F. J. Carmone, A. Kara, and S. H. Zanakis, "A Monte Carlo investigation of incomplete pairwise comparison matrices in AHP," Eur. J. Oper. Res., vol. 102, no. 3, pp. 538-553, 1997.

[35] I. Erol and J. W. Ferrell, "A methodology for selection problems with multiple, conflicting objectives and both qualitative and quantitative criteria," Int. J. Prod. Econ., vol. 86, no. 3, pp. 187-199, 2003.

[36] V. Bertsch, J. Geldermann, and O. Rentz, "Multidimensional monte carlo sensitivity analysis in multi-criteria decision support," in Proc. Symp. Autom. Syst. Based Human Skill Knowl., vol. 9, no. 1, 2006, pp. 141-146.

[37] T.-H. Hsu and F. F. Pan, "Application of monte carlo AHP in ranking dental quality attributes," Expert Syst. Appl., vol. 36, no. 2, pp. 2310-2316,2009.

[38] Z. P. Fan, Y. Liu, and B. Feng, "A method for stochastic multiple criteria decision making based on pairwise comparisons of alternatives with random evaluations," Eur. J. Oper Res., vol. 207, no. 2, pp. 906-915, 2010.

[39] I. Durbach, R. Lahdelma, and P. Salminen, "The analytic hierarchy process with stochastic judgements," Eur. J. Oper. Res., 2014.

[40] I. Basak, "Probabilistic judgments specified partially in the analytic hierarchy process," Eur. J. Oper. Res., vol. 108, no. 1, pp. 153-164, 1998.

[41] R. Banuelas and J. Antony, "Application of stochastic analytic hierarchy process within a domestic appliance manufacturer," J. Oper. Res. Soc., vol. 58, no. 1, pp. 29-38, 2007.

[42] K. N. Thompson, "Vendor profile analysis," J. Purchasing \& Mater. Manage., vol. 26, no. 1, p. 11, 1990.

[43] W.-N. Pi and C. Low, "Supplier evaluation and selection via taguchi loss functions and an ahp," Int. J. Adv. Manuf. Technol., vol. 27, no. 5-6, pp. 625-630, 2006.

[44] F. Zahedi, "Group consensus function estimation when preferences are uncertain," Oper. Res., vol. 34, no. 6, pp. 883-894, 1986.

[45] M. W. Herman and W. W. Koczkodaj, "A Monte Carlo study of pairwise comparison,” Inf. Process. Lett., vol. 57, no. 1, pp. 25-29, 1996.

[46] R. Aull-Hyde, S. Erdogan, and J. M. Duke, "An experiment on the consistency of aggregated comparison matrices in AHP," Eur. J. Oper. Res. vol. 171 , no. 1, pp. 290-295, 2006.

[47] J. C. Butler, J. S. Dyer, and J. Jia, "Using attributes to predict objectives in preference models," Decision Anal., vol. 3, no. 2, pp. 100-116, 2006.

[48] A. Ishizaka and M. Lusti, "How to derive priorities in AHP: A comparative study," Central Eur. J. Oper. Res., vol. 14, no. 4, pp. 387-400, 2006.

[49] P. T. Harker, "Alternative modes of questioning in the analytic hierarchy process," Math. Model., vol. 9, no. 3, pp. 353-360, 1987.

[50] A. Ishizaka, D. Balkenborg, and T. Kaplan, "Influence of aggregation and measurement scale on ranking a compromise alternative in AHP," J. Oper. Res. Soc., vol. 62, no. 4, pp. 700-710, 2010.

[51] F. J. Dodd and H. A. Donegan, "Comparison of prioritization techniques using interhierarchy mappings," J. Oper. Res. Soc., vol. 46, no. 4, pp. 492-498, 1995.

[52] D. Ma and X. Zheng, "9/9 $\pm 9 / 1$ scale method of AHP," in Proc. 2nd Int. Symp. Analytic Hierarchy Process, vol. 1, 1991, pp. 197-202.

[53] A. A. Salo and R. P. Hämäläinen, "On the measurement of preferences in the analytic hierarchy process," J. Multi-Criteria Decision Anal., vol. 6, no. 6, pp. 309-319, 1997.

[54] Google. (2013, Jan.). Google reader. [Online]. Available: http://www. google.com/reader/

[55] Likekhor. (2013, Jan). Welcome to: Likekhor.com. [Online]. Available: http://www.Likekhor.com 
[56] B. K. Kaye and T. J. Johnson, "Hot diggity blog: A cluster analysis examining motivations and other factors for why people judge different types of blogs as credible," Mass Commun. Soc., vol. 14, no. 2, pp. 236-263, 2011.

[57] G.-H. Tzeng, C.-H. Chiang, and C.-W. Li, "Evaluating intertwined effects in e-learning programs: A novel hybrid MCDM model based on factor analysis and DEMATEL," Expert Syst. Appl., vol. 32, no. 4, pp. 1028-1044, 2007.

[58] H. A. Simon, Administrative Behavior: A Study of Decision-making Processes in Administrative Organization. New York, NY, USA: Macmillan, 1976.

[59] J. J. Liou, G.-H. Tzeng, and H.-C. Chang, "Airline safety measurement using a hybrid model," J. Air Transp. Manage., vol. 13, no. 4, pp. 243-249, 2007.

[60] J. J. Liou and Y.-T. Chuang, "Developing a hybrid multi-criteria model for selection of outsourcing providers," Expert Syst. Appl., vol. 37, no. 5, pp. 3755-3761, 2010.

[61] J. J. Liou, "New concepts and trends of MCDM for tomorrow-in honor of Professor Gwo-Hshiung Tzeng on the occasion of his 70th birthday," Technol. Econ. Develop. Economy, vol. 19, no. 2, pp. 367-375, 2013.

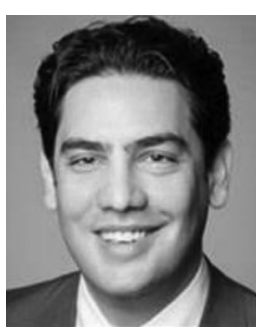

Niam Yaraghi received the Bachelor's degree in industrial engineering from the Isfahan University of Technology, Isfahan, Iran; the Master's degree in project management from the Royal Institute of Technology, Sweden; and the Ph.D. degree in management science from the University at Buffalo, Buffalo, NY, USA.

He is a Fellow in the Center for Technology Innovation, Brookings Institution, Washington, DC, USA. $\mathrm{He}$ is an expert on economics of healthcare information technology with a focus on health information exchange (HIE) systems. His research examines the network externalities in the healthcare market, and their effects on HIE adoption and usage. His current research interests include analyzing the outcomes of HIE use in reducing the costs and increasing the quality of healthcare services.

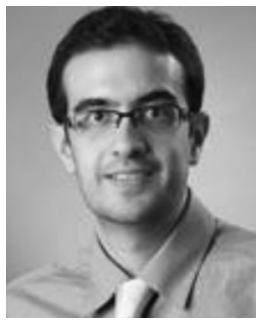

Pooya Tabesh received the B.S. degree in industrial engineering from the Isfahan University of Technology, Isfahan, Iran, in 2007, and the M.S. degree in industrial engineering from the University of Houston, Houston, TX, USA, in 2009. He is currently a Doctoral candidate in strategic management at the Bauer College of Business, University of Houston.

His main research interests include strategic decision making processes, behavioral decision theory, and decision support systems.

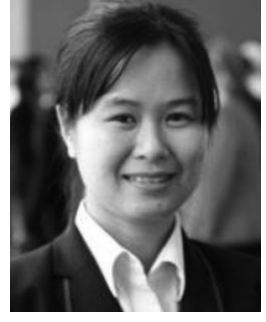

Peiqiu Guan received the B.S. degree in information and computing science from the Mathematics Department, Jinan University, Guangzhou, China, and the M.S. degree in risk management science from the Department of Statistics, Chinese University of Hong Kong, Hong Kong. She is currently working toward the Doctoral degree in the Department of Industrial and Systems Engineering, University at Buffalo, Buffalo, NY, USA.

Her research interests include mathematical modeling, game theory, operations research, validation experiments, and their applications to optimal defensive resource allocations in homeland security and disaster management.

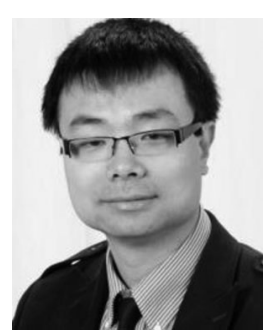

Jun Zhuang received the Bachelor's degree in industrial engineering from Southeast University, Nanjing, China, in 2002; the M.S. degree in agricultural economics from the University of Kentucky, Lexington, KY, USA, in 2004; and the Ph.D. degree in industrial engineering from the University of WisconsinMadison, Madison, WI, USA, in 2008

He has been a Faculty (Associate Professor, 2014 present; Assistant Professor, 2008-2014) of Industrial and Systems Engineering at the University at Buffalo, State University of New York, Buffalo, NY, USA. His long-term research goal is to integrate operations research, game theory, and decision analysis to improve mitigation, preparedness, response, and recovery for natural and man-made disasters. Other areas of his interest include applications to health care, sports, transportation and supply chain management, and sustainability. 